\title{
Chief Medical Officers back water fluoridation
}

\author{
The British Dental Association (BDA) \\ has welcomed the new joint statement ${ }^{1}$ \\ from all four UK Chief Medical Officers \\ (CMOs) highlighting the benefits of water \\ fluoridation in reducing tooth decay. \\ The BDA fully supports community water \\ fluoridation as a safe and effective public \\ health intervention, as part of a package \\ of measures to improve dental health, \\ where technically feasible and appropriate \\ for local needs. The Health and Care \\ Bill, currently before Parliament, is set to \\ simplify the rollout of the policy in England. \\ Dentist leaders are now urging all four UK \\ administrations to restate their positions on \\ water fluoridation in light of the statement. \\ Public Health England modelling shows \\ water fluoridation more than pays for itself \\ in the medium term, owing to reduction \\ in treatment need $-£ 1$ spent equates \\ to $£ 12.71$ savings in five years, rising to \\ $£ 21.98$ in ten. The BDA has stressed that \\ upfront investment by government is vital \\ to unlock these benefits. \\ In their statement, the four UK CMOs \\ recognise water fluoridation should be
}

seen as a complementary strategy, and not a substitute for regular dental check-ups and other effective methods of increasing fluoride use. The BDA backs a joined-up approach in which tried and tested policies like water fluoridation and supervised tooth brushing in early years settings are expanded, with parallel effort applied to rebuilding high street dental services.

Oral health inequality is anticipated to widen, given combination of unprecedented access problems, the suspension and ongoing disruption to public health programmes, and changes to dietary habits since the start of the pandemic. Over 30 million appointments have been lost in NHS dentistry since lockdown, in England alone.

Around 5.8 million people in England receive fluoridated water, the lion's share artificially added, but in some locations the appropriate level exists naturally within local water supplies.

BDA Chair Eddie Crouch said: 'Spending here will pay for itself, and Ministers need to show they are willing to seize the moment.

'We need a joined-up approach. COVID has left millions unable to access care, and deep inequalities are now set to widen. The four governments must double down on tried and tested policies while rebuilding the services millions depend on'.

The British Fluoridation Society (BFS) also welcomed the CMOs' statement. BFS Chair Dr Barry Cockcroft CBE said that the statement 'confirms that water fluoridation schemes are safe and effective and that they have a positive impact on tackling completely avoidable oral health inequalities in children and adults across the UK.

'The ability to strengthen tooth enamel and reduce cavities with the simple addition of fluoride to water is a powerful and cost-effective tool, along with other preventive measures'.

\section{Reference}

1. Department of Health \& Social Care. Independent report-Statement on water fluoridation from the UK Chief Medical Officers. 2021. Available at: https://www.gov.uk/government/publications/ water-fluoridation-statement-from-the-uk-chiefmedical-officers/statement-on-water-fluoridation from-the-uk-chief-medical-officers (accessed 28 September 2021).

\section{Updated Delivering better oral health published}

Public Health England has published the latest edition of the Delivering better oral health $(\mathrm{DBOH})$ toolkit. ${ }^{1} \mathrm{DBOH}$ is an evidence-based toolkit intended to support dental teams in improving their patients' oral and general health.

The fourth edition of the toolkit, which has been developed with the support of the four UK Chief Dental Officers, is intended to be relevant to all dental team members as it:

- Supports primary care dental teams to routinely promote oral and general health

- Facilitates the provision of optimal care, advice and support for patients in achieving and maintaining good oral health

- Is an educational resource for dental schools, postgraduate deaneries, and other providers and commissioners of dental teaching

- Is equally appropriate to dental specialists and their teams
- May be used across health and social care

- Allows commissioning bodies to implement preventive pathways of care

- Will be supported by resources to facilitate continuing professional development.

Whilst the guidance seeks to ensure a consistent UK-wide approach to prevention of oral diseases, some differences in operational delivery and organisational responsibilities may apply in Wales, Northern Ireland and England. In Scotland, the guidance will be used to inform oral health improvement policy.

\section{Reference}

Public Health England, Department of Health and Social Care, NHS England and NHS Improvement. Delivering better oral health: an evidence-based toolkit for prevention. Updated 21 September 2021. Available at: https://www.gov.uk/government/publications/ delivering-better-oral-health-an-evidence-basedtoolkit-for-prevention (accessed 28 September 2021).
Dentists can now pay their ARF in instalments

Dentists can now sign up to pay their Annual Retention Fee (ARF) in instalments, following the successful introduction of the scheme for dental care professionals (DCPs) earlier this year.

The General Dental Council (GDC) introduced the new pay by instalments option to support dental professionals who pay their own fee by enabling them to spread the cost over the year. For dentists, this is four direct debits of $£ 170$.

To take advantage of the option this year, dentists need to log in to eGDC and select the quarterly direct debit option no later than 31 October 2021.

Where dentists hold specialist titles, the annual fee of $£ 72$ per title will also be collected in the first direct debit. Visit https://bit.ly/3m7YMD2. 